\title{
Aharonov-Bohm effect in a singly connected point contact
}

\author{
$\sim B-1$ \\ P. H. M. van Loosdrecht, ${ }^{* \dagger}$ C. W. J. Beenakker, H. van Houten, ${ }^{\ddagger}$ and J. G. Williamson \\ Philips Research Laboratories, 5600 JA Eindhoven, The Netherlands \\ B. J. van Wees and J. E. Mooij \\ Department of Applied Physics, Delft University of Technology, $2600 \mathrm{GA}$ Delft, The Netherlands \\ C. T. Foxon and J. J. Harris \\ Philips Research Laboratories, Redhill, United Kingdom
}

(Received 4 August 1988)

\begin{abstract}
We report the discovery of an oscillation in the low-temperature magnetoresistance of a point contact in the two-dimensional electron gas of a GaAs- $\mathrm{Al}_{x} \mathrm{Ga}_{1-x} \mathrm{As}$ heterostructure. The oscillation is periodic in the magnetic field and is reminiscent of the Aharonov-Bohm effect in rings, although the geometry is singly connected. A possible mechanism for this quantum interference effect is tunneling between edge states across the point contact at the potential step at the entrance and the exit of the constriction.
\end{abstract}

Aharonov-Bohm (AB) magnetoresistance oscillations are a fundamental manifestation of the influence of a magnetic field on the phase of the electron wave function. The effect has been studied extensively in metal rings and cylinders, ${ }^{1}$ and has recently also been seen (with a much larger amplitude) in rings defined in the two-dimensional electron gas (2D EG) of a $\mathrm{GaAs}-\mathrm{Al}_{x} \mathrm{Ga}_{1-x} \mathrm{As}$ heterostructure. ${ }^{2,3}$ In these experiments oscillations in the resistance of the ring are observed as a function of the applied perpendicular magnetic field $B$. The oscillations are periodic in $B$, with a fundamental period $\Delta B=h / e A$ determined by the area $A$ of the ring. Their origin is the fieldinduced phase difference between the two paths (one clockwise, one counterclockwise) which take an electron from one side of the ring to the other.

In this paper, we report the observation of periodic oscillations in the magnetoresistance of a narrow and short constriction (point contact) connecting two broad regions in a $2 \mathrm{D}$ EG. The effect is reminiscent of the AB effect in rings, but occurs in a singly connected geometry. The period of the oscillations is constant within $5 \%$ over the field range from 2 to $8 \mathrm{~T}$ where the effect is observed. A splitting of the peaks develops as the field resolves the spin degeneracy. We interpret the oscillations as a quantum interference effect in view of the fact that they disappear on increasing current or temperature and are absent in a parallel magnetic field. As a possible mechanism, which can explain the remarkable periodicity of the oscillations, we propose a novel AB effect associated with the flux enclosed by two "tunneling paths"- rather than by the two classical paths in a ring. We base our argument on the study of tunneling problems in high magnetic fields by Jain and Kivelson. ${ }^{4}$ Related mechanisms, based on circulating edge currents, have been considered for $\mathrm{AB}$ effects in small conductors. ${ }^{5-7}$ An experimental feature which is not understood is that the amplitude of the oscillations is changed on reversing the magnetic field.

Studies of quantum transport through constrictions have revealed a wealth of interesting new phenomena. ${ }^{8-11}$
Chang et al. ${ }^{12}$ have observed aperiodic fluctuations superimposed on quantum Hall $(\mathrm{QH})$ plateaus. van Wees et al. ${ }^{13}$ and Wharam et al. ${ }^{14}$ have fabricated constrictions with variable dimensions much smaller than the mean free path, and of the order of the Fermi wavelength. It was discovered that the conductance of these point contacts is quantized in units of $2 e^{2} / h$ without a magnetic field. Upon application of a perpendicular field a smooth transition to the QH plateaus is observed. Electron focusing in a magnetic field has been realized using two such quantum point contacts, and shows fine structure due to the phase coherence of the focused electrons. ${ }^{15}$ The magnetoresistance oscillations reported below were observed in a point contact device ${ }^{13,15}$ fabricated on a high-mobility GaAs- $\mathrm{Al}_{x} \mathrm{Ga}_{1-x} \mathrm{As}$ heterostructure (sheet carrier concentration $n_{0}=3.6 \times 10^{15} \mathrm{~m}^{-2}$, and mean free path $8.5 \mu \mathrm{m}$ ). The point contact is defined electrostatically by means of a split gate (opening $250 \mathrm{~nm}$ ) on top of the heterostructure (Fig. 1, inset). By applying a negative voltage on the gate, a narrow and short constriction is created in the $2 \mathrm{D}$ EG. The 2D EG directly under the gate is depleted at gate voltages $V_{g} \lesssim-0.6 \mathrm{~V}$, and the point contact is fully pinched off at $V_{g} \lesssim-2.4 \mathrm{~V}$. A low-frequency ac lock-in technique with voltages below $5 \mu \mathrm{V}$ was used to measure the two-terminal resistance $R_{2 t}$ of the point contact. Results are shown in Figs. 1 and 2.

The bottom curve in Fig. 1(b) gives $R_{2 t}$ for $V_{g}=0 \mathrm{~V}$, when the 2D EG below the gate is not depleted. This two-terminal resistance measurement shows the characteristic superposition of Shubnikov-de Haas ( $\mathrm{SdH}$ ) oscillations (periodic in $1 / B$ ) and $\mathrm{QH}$ plateaus at values $h / e^{2} i \simeq 26 \mathrm{k} \Omega / i$ (coinciding with minima of the $\mathrm{SdH}$ oscillations). Around zero field, a negative magnetoresistance peak appears, originating in the source and drain contacts. ${ }^{15}$ The top curve in Fig. 1 (a) gives the magnetoresistance of the point contact (for $V_{g}=-1.65 \mathrm{~V}$ ) at the relatively high temperature of $1 \mathrm{~K}$. The density $n_{c}$ in the point contact region is reduced by applying a gate voltage, ${ }^{13}$ which results in a shift of the $\mathrm{QH}$ plateaus to lower 

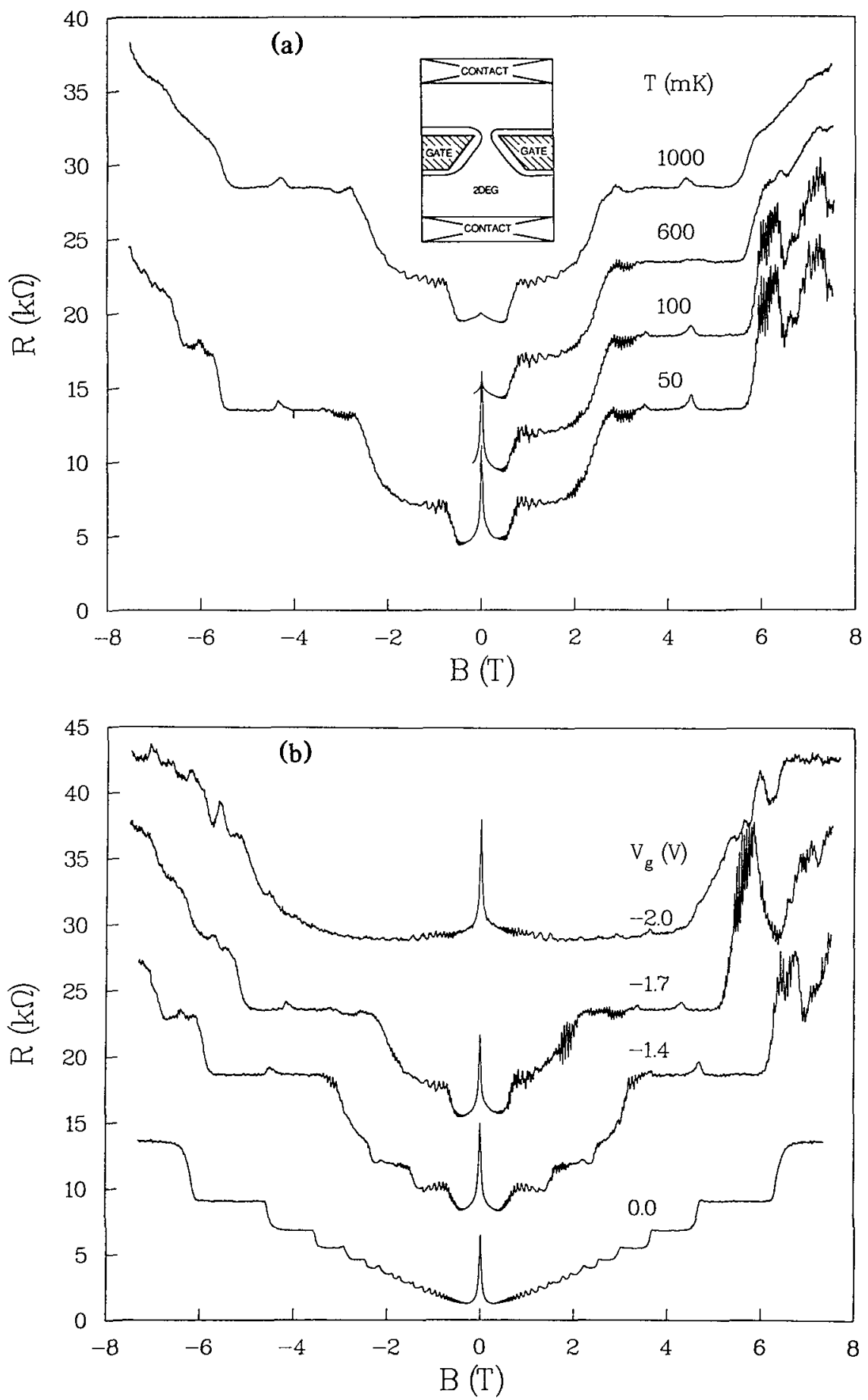

FIG. 1. Two-terminal magnetoresistance of a point contact for a series of temperatures and gate voltages $\left[V_{g}=-1.65 \mathrm{~V}\right.$ in (a), and $T=50 \mathrm{mK}$ in (b)]. The second, third, and fourth curve from the bottom have offsets of, respectively, 5,10 , and $15 \mathrm{k} \Omega$. The inset in (a) shows the device geometry.

fields. From the location of the plateaus we estimate $n_{c}=1.5 \times 10^{15} \mathrm{~m}^{-2}$ for $V_{g}=-1.65 \mathrm{~V}$. Note that the $\mathrm{QH}$ plateaus (determined by $n_{c}$ ) no longer coincide with the minima of the $\mathrm{SdH}$ oscillations (which originate in the broad 2D EG, and have a periodicity determined by $n_{0}$ ). At low fields, $R_{2 t}$ is determined by the number $N_{c}$ of occupied subbands in the constriction, according to ${ }^{13,14}$ $R_{2 t}=h / 2 e^{2} N_{c}$. For a square-well confining potential we have $N_{c} \simeq k_{F} W / \pi$, where $W$ is the constriction width, and $k_{F} \equiv\left(2 \pi n_{c}\right)^{1 / 2}$ is the Fermi wave vector in the constric- tion. We thus estimate $W \simeq 90 \mathrm{~nm}$ for $V_{g}=-1.65 \mathrm{~V}$.

Upon lowering the temperature, reproducible large amplitude oscillations develop in $R_{2 t}$ for $B \gtrsim 2 \mathrm{~T}$. In contrast with the $\mathrm{SdH}$ oscillations (periodic in $1 / B$ ), these oscillations are periodic in $B$ itself. The periodicity for $V_{g}$ $=-1.65 \mathrm{~V}$ is $\Delta B=0.056 \pm 0.003 \mathrm{~T}$ over the field range from 2 to $8 \mathrm{~T}$. The oscillations occur predominantly between the QH plateaus, and not on the plateaus themselves. No periodic oscillations are observed at the highest negative gate voltages [top curve in Fig. 1(b)]. No effect 
$5 \quad 525456586 \quad 626466687 \quad 7274$

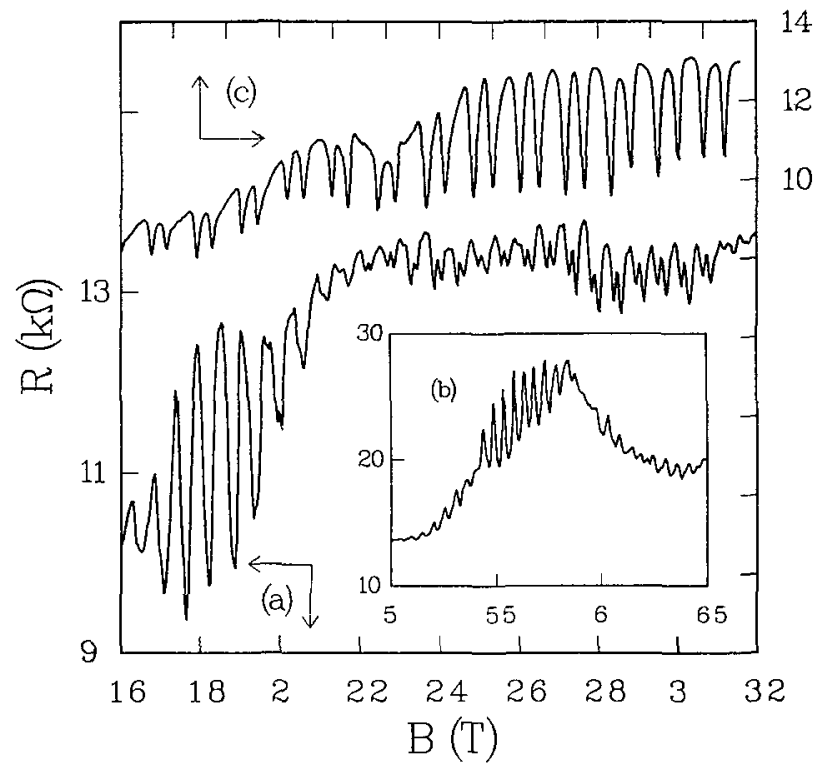

FIG. 2. Curves $a$ and $b$ are close-ups of the curve for $V_{g}=-1.7 \mathrm{~V}$ in Fig. 1(b). Curve $c$ was measured three months earlier on the same device (note the different field scale, due to a change in electron density in the constriction).

is found if $B$ is parallel to the 2D EG. In the range of gate voltages where the oscillations occur, $\Delta B$ is insensitive within $10 \%$ to changes in $V_{g}$. This is much less than the estimated relative variations of $n_{c}$ and $W$. A close-up of the oscillations shows the development of a splitting of the peaks between the $i=4$ and the $i=2$ spin-degenerate $\mathrm{QH}$ plateaus [Fig. 2(a)]. The peak separation increases approximately linearly with $B$, indicating that the two components of the peak have a slightly different (a few percent) but approximately constant periodicity. Only a single peak is seen at higher fields, see the transition from $i=2$ to $i=1$ in Fig. 2(b). Large changes in the device properties are obtained if it is brought to room temperature and then cooled again, and this has a much stronger effect on $\Delta B$ than variations in the gate voltage. This is illustrated by Fig. 2(c), obtained three months earlier than 2(a). In curve $c, \Delta B=0.18 \mathrm{~T}$ is three times larger than in curve $a$. Both sets of data show the peak splitting between the $i=4$ and $i=2$ plateaus, which occurs at higher fields in curve $c$ because of an increase in $n_{c}$ of about a factor of 2. The periodic oscillations have been observed in only one of several point contact devices available. We should emphasize, however, the overall reproducibility of the effect with its characteristic peak splitting over a period of several months, as evidenced by Fig. 2 .

One sees from Fig. 1 that the periodic oscillations have a much smaller amplitude in reverse fields (although the periodicity is the same). Note also that a slow modulation of $R_{2 t}$ around $B=7 \mathrm{~T}$ is observed in one field direction only. The generalized reciprocity relation derived by Büttiker ${ }^{16}$ for nonlocal phase coherent resistance measurements predicts that two-terminal resistances should be symmetric, $R_{2 t}(B)=R_{2 t}(-B)$, provided no magnetic impurities are present. We verified the symmetry of $R_{2 t}$ (to within $10 \%$ ) in other points contact devices, which contained large magnetoresistance fluctuations-but without a well-defined periodicity.

We now discuss the tunneling mechanism for $\mathrm{AB}$ oscillations, illustrated in Fig. 3. The split gate (shaded) both confines the electrons laterally and reduces their density by raising the bottom of the conduction band relative to the broad 2D EG regions. As a consequence, the electrostatic potential has a saddle form. The classical motion in a strong magnetic field is along equipotentials, which are shown schematically (arrows point in the direction of motion, determined by the potential gradient). The energy of the equipotential is the guiding center energy $E_{G}$, which for an electron in the $n$th Landau level is given by

$$
E_{G}=E_{F}-\left(n-\frac{1}{2}\right) \hbar \omega_{c} \pm \frac{1}{2} g \mu_{B} B .
$$

Here $E_{F}$ is the Fermi energy (which may depend on $B$ due to pinning at the Landau levels), $\omega_{c}=e B / m$ is the cyclotron frequency, and $g \mu_{B} B$ is the Zeeman spin splitting. Tunneling corresponds to motion across the equipotentials. An electron which enters the constriction at $a$ can be reflected back into the broad region by tunneling to the opposite edge, either at the potential step at the entrance of the constriction (from $a$ to $b$ ) or at its exit (from $d$ to $c)$. These two tunneling paths acquire an $\mathrm{AB}$ phase difference ${ }^{4}$ of order $e B A / \hbar$ (where $A$ is the enclosed area $a b c d$ ), leading to magnetoresistance oscillations of periodicity $h / e A$. For a well-defined area $A$, the potential $V(x, y)$ should vary rapidly over a short distance. The possibility in principle of such an effect can be demonstrated by a simple model calculation. Following Jain and Kivelson, ${ }^{17}$ we have studied the saddle potential $V(x, y)=\frac{1}{2} m \omega_{0}^{2} y^{2}+V_{B}(x)$, where $V_{B}(x)$ is a rectangular barrier of height $V_{0}$ extending over a length $L$. The transmission probability $T$ in the large- $B$ limit is ${ }^{17}$ (for $\left.E_{G}>V_{0}\right)$

$$
r^{-1}=1+\frac{V_{0}^{2}}{4 E_{G}\left(E_{G}-V_{0}\right)} \sin ^{2}\left(e B L W_{G} / 2 \hbar\right),
$$

and shows $\mathrm{AB}$ oscillations determined by the constriction length $L$ and its width $W_{G} \equiv 2\left[\left(E_{G}-V_{0}\right) 2 / m \omega_{0}^{2}\right]^{1 / 2}$ at the guiding center energy.

The above mechanism can account for the observed periodicity: Using $\Delta B \sim h / e A$, experimental constriction areas $A$ in the range from $(250 \mathrm{~nm})^{2}$ to $(150 \mathrm{~nm})^{2}$ are de-

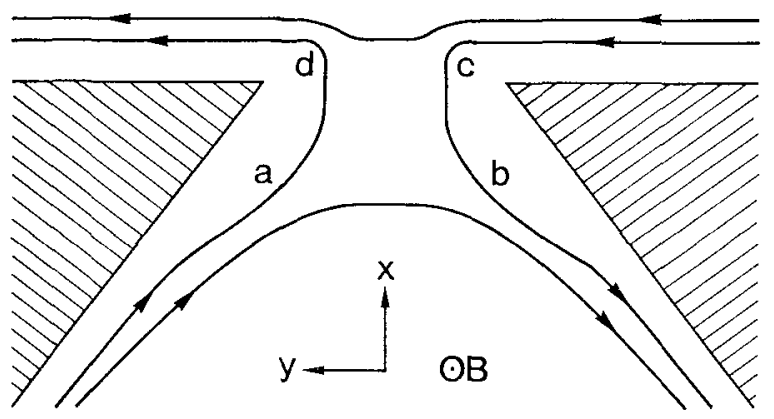

FIG. 3. Illustration of the tunneling mechanism for $A B$ oscillations in the point contact magnetoresistance, discussed in the text. 
rived, in accord with estimates for its width (from 100 to $200 \mathrm{~nm})$ and length ${ }^{18}(L \gtrsim W)$. The experimental insensitivity of $\Delta B$ to gate voltage changes is consistent with the increasing length of the constriction as its width is reduced. The predominance of the oscillations between the $\mathrm{QH}$ plateaus can be understood in terms of Eq. (2), since at these fields we expect a nearly depopulated Landau level with guiding center energy $E_{G}$ close to the potential step $V_{0}$ in the constriction, so that the oscillations in $T$ are large. The tunneling $\mathrm{AB}$ effect requires that $W$ is large compared to the magnetic length $l_{m} \equiv(\hbar / e B)^{1 / 2}$, to permit the spatial separation in edge states of electrons moving in opposite directions. Indeed, the periodic oscillations are absent in the narrowest constrictions [top curve in Fig. 1(b)]. Spin splitting of Landau levels causes spin-up and spin-down electrons to move along equipotentials enclosing slightly different areas [cf. Eq. (1)], thus explaining the experimental splitting of the oscillation peaks at higher fields. This is a striking feature of the effect, which might also be observable in 2D EG rings if studied in a tilted magnetic field (in order to avoid the high field suppression of the $\mathrm{AB}$ effect ${ }^{19}$ ). The above mechanism does not explain the observed asymmetry on reversing the magnetic field. This fact, combined with the sensitivity of the periodicity of the oscillations to temperature cycling and the absence of the effect in other devices of the same design, suggests that magnetic impurity scattering may play a role. We intend to study the tunneling $A B$ effect further in specially designed geometries.

We thank M. E. I. Broekaart for assistance with the experiments, and C. J. P. M. Harmans, J. A. Pals, and M. F. $\mathrm{H}$. Schuurmans for support. We acknowledge the facilities offered by the Delft Centre for Submicron Technology and the financial support from the Stichting voor Fundamenteel Onderzoek der Materie.
*Also at the Eindhoven University of Technology, $5600 \mathrm{MB}$ Eindhoven, The Netherlands.

†resent address: Research Institute for Materials, University of Nijmegen, 6525 ED Nijmegen, The Netherlands.

†resent address: Philips Laboratories, Briarcliff Manor, NY 10510.

${ }^{1}$ See reviews by S. Washburn and R. A. Webb, Adv. Phys. 35, 375 (1986); A. G. Aronov and Yu. V. Sharvin, Rev. Mod. Phys. 59, 755 (1987).

${ }^{2}$ G. Timp, A. M. Chang, J. E. Cunningham, T. Y. Chang, P. Mankiewich, R. Behringer, and R. E. Howard, Phys. Rev. Lett. 58, 2814 (1987).

${ }^{3}$ C. J. B. Ford, T. J. Thornton, R. Newbury, M. Pepper, H. Ahmed, C. T. Foxon, J. J. Harris, and C. Roberts, J. Phys. C 21, L325 (1988)

${ }^{4}$ J. K. Jain and S. Kivelson, Phys. Rev. B 37, 4111 (1988); see also H. A. Fertig and B. I. Halperin, ibid. 36, 7969 (1987).

${ }^{5}$ E. N. Bogachek and G. A. Gogadze, Zh. Eksp. Teor. Fiz. 63, 1839 (1972) [Sov. Phys. JETP 36, 973 (1973)]; N. B. Brandt, D. V. Gitsu, A. A. Nikolaeva, and Ya.G. Ponomarev, ibid. 72, 2332 (1977) [ ibid. 45, 1226 (1977)].

${ }^{6}$ Y. Isawa, Surf. Sci. 170, 38 (1986).

${ }^{7}$ U. Sivan, Y. Imry, and C. Hartzstein (unpublished).

${ }^{8}$ J. R. Kirtley, Z. Schlesinger, T. N. Theis, F. P. Milliken, S. L. Wright, and L. F. Palmateer, Phys. Rev. B 34, 5414 (1986).

${ }^{9}$ L. Bliek, E. Braun, G. Hein, V. Kose, J. Niemeyer, G Weimann, and W. Schlapp, Semicond. Sci. Technol. 1, 110 (1986).

${ }^{10}$ H. van Houten, C. W. J. Beenakker, P. H. M. van Loosdrecht, T. J. Thornton, H. Ahmed, M. Pepper, C. T. Foxon, and J. J.
Harris, Phys. Rev. B 37, 8534 (1988).

${ }^{11}$ M. A. Kastner, S. B. Field, J. C. Licini, and S. L. Park, Phys. Rev. Lett. 60, 2535 (1988).

${ }^{12}$ A. M. Chang, G. Timp, T. Y. Chang, J. E. Cunningham, P. M. Mankiewich, R. E. Behringer, and R. E. Howard (unpublished).

${ }^{13}$ B. J. van Wees, H. van Houten, C. W. J. Beenakker, J. G. Williamson, L. P. Kouwenhoven, D. van der Marel, and C. T. Foxon, Phys. Rev. Lett. 60, 848 (1988); B. J. van Wees et al., Phys. Rev. B 38, 3625 (1988).

${ }^{14}$ D. A. Wharam, T. J. Thornton, R. Newbury, M. Pepper, H. Ahmed, J. E. F. Frost, D. G. Hasko, D. C. Peacock, D. A. Ritchie, and G. A. C. Jones, J. Phys. C 21, L209 (1988).

${ }^{15} \mathrm{H}$. van Houten, B. J. van Wees, J. E. Mooij, C. W. J. Beenakker, J. G. Williamson, and C. T. Foxon, Europhys. Lett. 5, 721 (1988); C. W. J. Beenakker, H. van Houten, and B. J. van Wees, ibid. (to be published).

${ }^{16}$ M. Büttiker, Phys. Rev. Lett. 57, 1761 (1986); IBM J. Res. Dev. 32, 317 (1988).

${ }^{17}$ J. K. Jain and S. A. Kivelson, Phys. Rev. B 37, 4276 (1988).

${ }^{18}$ The observation of plateaus in $R_{2 t}$ at $B=0$ indicates $L \gtrsim W$, so that a $1 D$ density of states applies in the constriction region. Relatively large values of $L$ also follow from an analysis of modulations in the zero-field conductance as a function of $V_{g}$, in terms of interferences between electrons reflected at the entrance and the exit of the constriction [B. J. van Wees et al. (unpublished)].

${ }^{19}$ G. Timp, P. M. Mankiewich, P. deVegvar, R. Behringer, J. E. Cunningham, R. E. Howard, H. U. Baranger, and J. K. Jain (unpublished); J. K. Jain, Phys. Rev. Lett. 60, 2074 (1988). 